\title{
Disposable Solid-State pH Sensor Using Nanoporous Platinum and Copolyelectrolytic Junction ${ }^{\dagger}$
}

\author{
Jongmin Noh, Sejin Park, ${ }^{\ddagger}$ Hee Chan Kim,,${ }^{\S, *}$ and Taek Dong Chung, \\ Interdisciplinary Program, Biomedical Engineering Major, Graduate School, Seoul National University, \\ Seoul 152-742, Korea \\ ${ }^{\ddagger}$ Nomadien Corporation, B-206, SK Twintech Tower, Gasan-dong, Geumcheon-gu, Seoul 153-773, Korea \\ ${ }^{\S}$ Institute of Medical \& Biological Engineering, Medical Research Center and Department of Biomedical Engineering, \\ College of Medicine, Seoul National University, Seoul 110-799, Korea. ${ }^{*}$ E-mail: hckim@snu.ac.kr \\ ${ }^{\#}$ Department of Chemistry, Seoul National University, Seoul 151-747, Korea. ${ }^{*}$-mail: tdchung@snu.ac.kr \\ Received August 30, 2010, Accepted September 13, 2010
}

\begin{abstract}
A disposable solid-state $\mathrm{pH}$ sensor was realized by utilizing two nanoporous $\mathrm{Pt}(\mathrm{npPt})$ electrodes and a copolyelectrolytic junction. One nanoporous Pt electrode was to measure the $\mathrm{pH}$ as an indicating electrode $(\mathrm{pH}-\mathrm{IE})$ and the other assembled with copolyelectrolytic junction was to maintain constant open circuit potential $\left(E_{\mathrm{oc}}\right)$ as a solid-state reference electrode (SSRE). The copolyelectrolytic junction was composed of cationic and anionic polymers immobilized by photo-polymerization of $N, N^{\prime}$-methylenebisacrylamide, making buffered electrolytic environment on the SSRE. It was expected to make. The nanoporous Pt surrounded by a constant $\mathrm{pH}$ excellently worked as a solid state reference electrode so as to stabilize the system within $30 \mathrm{~s}$ and retain the electrochemical environment regardless of unknown sample solutions. Combination between the SSRE and the $\mathrm{pH}-\mathrm{IE}$ commonly based on nanoporous $\mathrm{Pt}$ yielded a complete solid-state $\mathrm{pH}$ sensor that requires no internal filling solution. The solid state $\mathrm{pH}$ sensing chip is simple and easy to fabricate so that it could be practically used for disposable purposes. Moreover, the solid-state $\mathrm{pH}$ sensor successfully functions in calibration-free mode in a variety of buffers and surfactant samples.
\end{abstract}

Key Words: Nanoporous platinum, Copolyelectrolyte junction, Solid-state $\mathrm{pH}$ sensor, Disposable $\mathrm{pH}$ sensor, Calibration-free $\mathrm{pH}$ sensor

\section{Introduction}

A pH measuring device has been essentially required for various purposes, and a few types of $\mathrm{pH}$ sensors have been developed to meet such demands. However, most of the $\mathrm{pH}$ sensors, which are currently available and being used in practice, are glass membrane combination electrodes. They are fragile, expensive and bulky so as to be handled with care, always kept in the storage solution when not used, and mandatorily calibrated before use.

Solid-state $\mathrm{pH}$ sensor has been one of the potential alternatives to overcome these drawbacks of glass membrane combination electrodes. ${ }^{1-10}$ For example, the hydrogen ion sensitive fieldeffect transistors $\left(\mathrm{H}^{+}\right.$-ISFET), ${ }^{1}$ metal oxides (iridium oxide, ${ }^{2-4}$ platinum oxide ${ }^{5}$ ), and $\mathrm{H}^{+}$-selective electrodes based on poly (vinylchloride)/polyurethane membrane containing tridodecylamine ${ }^{6,7}$ were proposed as new $\mathrm{pH}$-indicating electrodes $(\mathrm{pH}-$ IEs). These solid state $\mathrm{pH}$-IEs exhibited good performance, but they still use reference electrodes (REs) such as $\mathrm{Ag} / \mathrm{AgCl} /$ $\mathrm{KCl}(a q)$ with filling solution and liquid junction that hamper miniaturization and mass-production of the whole $\mathrm{pH}$ sensor system. For the disposable purpose of point-of-care test (POCT), the $\mathrm{pH}$ sensors are often prepared on a chip, which could be fully miniaturized and friendly to mass-production. For this reason, the solid-state reference electrode (SSRE) without filling solution or liquid junction has been studied to be integrated in

${ }^{\dagger}$ This paper is dedicated to Professor Hasuck Kim for his outstanding contribution to electrochemistry and analytical chemistry. a microchip. ${ }^{6,7}$ Many attempts have been made to develop successful SSRE by covering $\mathrm{Ag} / \mathrm{AgCl}$ with electrolyte-doped polymer, ${ }^{8,9}$ poly(vinylchloride) membranes with plasticizer, lipophilic salt, or cation or anion exchanger, ${ }^{10} \mathrm{KCl}$ containing hydrogel layer, ${ }^{6}$ polyurethane, ${ }^{7}$ or electrolyte loaded polymers. ${ }^{11,12}$ However, the present SSREs are not sufficiently reproducible and need long stabilization period.

Reportedly, nanoporous Pt (npPt) electrode shows far better $\mathrm{pH}$ sensing performance than flat Pt electrode ${ }^{5}$ by virtue of low polarizability resulting from extremely enlarged electrode surface area. In addition, npPt can act as a good RE when it is in buffer solutions. $^{13}$

In this contribution, a new type of SSRE is proposed by covering $\mathrm{npPt}$ with polyelectrolytes which maintain a constant $\mathrm{pH}$ like a pseudo buffer. The polyelectrolyte mixture consisting of weak acidic or basic functional groups such as poly(acrylic acid) (PAA), poly(ethyleneimine) (PEI), and their copolyelectrolyte (PEI-co-PAA) were suggested and tested. Harnessing the proposed SSRE, a solid-state $\mathrm{pH}$ sensor was successfully prepared on an alumina substrate.

\section{Experimental}

Reagents. Sodium tetraborate, boric acid, citric acid, sodium citrate, tert-octylphenoxypolyethoxyethanol (Triton X-100), hydrogen hexachloroplatinate hydrate (HCPA), polyacrylic acid (PAA, \#323667, average Mw 1800), polyethyleneimine (PEI, \#482595, average Mw 1300, 50 wt \% in water), 2-hydroxy-4'-(2-hydroxyethoxy)-2-methylpropiophenone (photo- 
initiator), $N, N^{\prime}$-methylenebisacrylamide (cross linker), and dimethylsulphoxide (DMSO) were purchased from Sigma. $\mathrm{NaH}_{2} \mathrm{PO}_{4}(98 \%), \mathrm{Na}_{2} \mathrm{HPO}_{4}(98 \%)$, and $\mathrm{KCl}(99.5 \%)$ were purchased from Junsei Chemical.

Fabrication of solid-state $\mathrm{RE}$ and $\mathrm{pH}$ sensor. Au conducting patterns were prepared on alumina substrate by subsequent screen-printing of Au paste and dielectric silica (as insulating coating) followed by thermal curing at $800{ }^{\circ} \mathrm{C}$. The npPt was electroplated on the Au conducting pattern and the real surface area of npPt was measured where a conversion factor was 210 $\mu \mathrm{C} \mathrm{cm}{ }^{-2}$, following the same method reported previously. ${ }^{5,14}$ The electroplated npPt was coated with polyphenol membrane and used as $\mathrm{pH}-\mathrm{IE}$. $^{5}$ The channel and chambers were fabricated by a conventional computer numerical control machine (Hwacheon Inc., Korea). The polyacrylate plate, briefly called acryl cover, was dipped in trimethylailylmethylamine, TMSMA, solution (Sigma) and kept in a vacuum chamber for $30 \mathrm{~min}$. An alumina substrate with npPt and a cover plate with a microchannel and chambers were bonded to each other with silicone double-sided adhesive tape (Youngwoo, Korea) by applying thermal pressure (Seoul system, Korea).

The polyelectrolytic (PE) junction in this study is a polymeric salt bridge in which a constant $\mathrm{pH}$ and high ionic conductivity are maintained. Two polymers with weak base and acid functional groups were employed for this 'PE junction', PEI and PAA. The proposed PE junction was fabricated according to previously reported method with some modification. ${ }^{15}$ A solution containing PEI or PAA (50 wt \%) and photo-initiator (2 wt \%) was prepared using DI water (solution A). To prepare PEI-co-PAA junction, both PEI and PAA were used in 1:1 (wt) ratio. The other solution containing cross linker (5 wt \%) was prepared using 1:1 (vol) solvent mixture of DMSO and DI water (solution B). A solution for photo-polymerization was prepared by mixing solution $A$ and $B$ in 3:1 (vol) ratio. The photo-polymerization was performed by aligning a mask to let only the PE microchannel and RE chamber exposed, and then by illuminating UV light ( $\left.365 \mathrm{~nm}, 16 \mathrm{~mW} \mathrm{~cm}^{-2}, 60 \mathrm{~s}\right)$. After photo-polymerization, the residual solution was removed and rinsed twice with $1 \mathrm{M} \mathrm{KCl}$ and $\mathrm{DI}$ water.

\section{Results and Discussion}

Figure 1 illustrates the structure of the solid-state $\mathrm{pH}$ sensor proposed in this study. There are a $\mathrm{pH}-\mathrm{IE}$ and a RE on an alumina substrate, between which a PE junction in the PE microchannel are present. The whole chip was constructed by assembling of the alumina substrate and the mechanically machined cover plate. npPts were electroplated on the two identical circular areas ( $2 \mathrm{~mm}$ in diameter) of the Au conducting patterns to be used as a $\mathrm{pH}$-IE and a RE. The npPt for $\mathrm{pH}-\mathrm{IE}$ was exposed to sample solution in the sampling chamber (4 $\mathrm{mm}$ in diameter). The other npPt was assembled with PE junction and used as a SSRE.

The role of the PE junction can be explained by imagining a polyelectrolyte plug between a pair of electrodes. The schematics in Figure 2 show three cases of PE junctions made of cationic PEI $\left(\mathrm{p} K_{\mathrm{a}}=5.5\right)$, anionic PAA $\left(\mathrm{p} K_{\mathrm{a}}=4.3\right)$ or mixed PEI-co-PAA layer. When PEI or PAA fills in the RE chamber and the PE
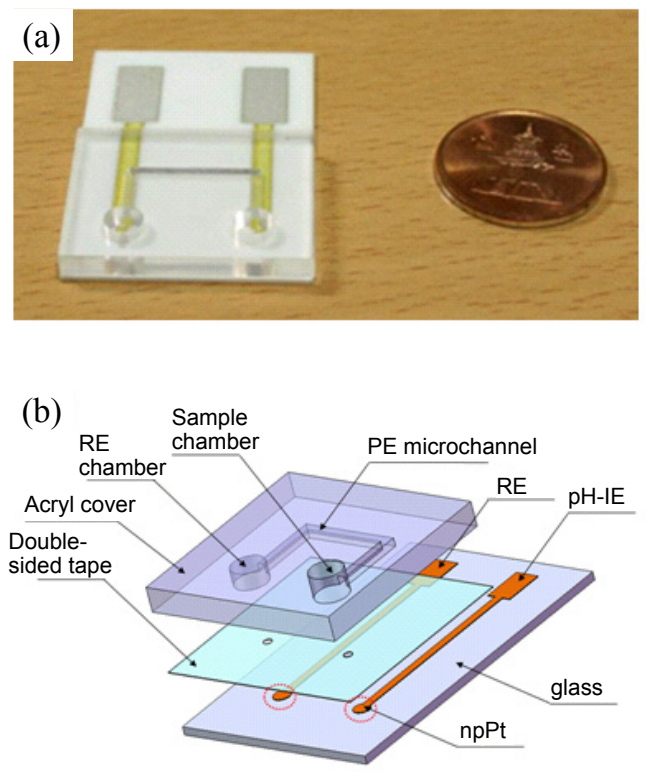

(c)

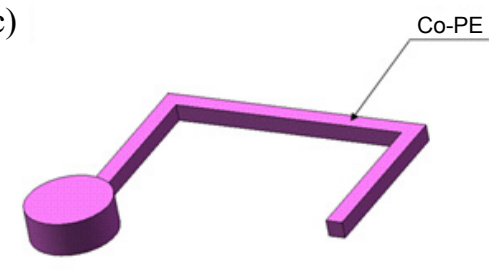

Figure 1. (a) Photogragh of the proposed solid-state $\mathrm{pH}$ sensor. (b) Schematic diagrams of the solid-state $\mathrm{pH}$ sensor and (c) the RE chamber and PE microchannel filled with the polyelectrolytic mixture, 'PE junction'.

microchannel, the $\mathrm{pH}$ in the $\mathrm{PE}$ junction is determined by the acid-base equilibrium of the polymeric functional groups. If PEI or PAA is filled in the microchannel and RE chamber, the PE plug allows only flow of counter ions due to electrostatic repulsion. The counter ions from the unknown sample solution may modify the conductivity of the PE junction and even contaminate the RE surface. Such an effect appears as time passes after the system is exposed to the sample solution. As a consequence, the $\mathrm{pH}$ near the npPt of RE may be affected by the sample solution as shown Figure 2(a) and 2(b). In addition, it should be noted that the $\mathrm{pH}$ in the $\mathrm{RE}$ chamber is maintained around $\mathrm{p} K_{\mathrm{a}}$ of the polymeric functional groups. So the buffer capacity may be insufficient at a $\mathrm{pH}$ which is far from the polymeric $\mathrm{p} K_{\mathrm{a}}$.

These problems are expected to be alleviated by mixing two acidic and basic polymers. The mixed PEI-co-PAA has both cationic and anionic sites as stationary phase, which needs only a limited amount of mobile ions to maintain the potentiometric circuit. Minimizing the ion transport through PEI-co-PAA junction and retaining sufficient ionic conductivity, the potential drop across $\mathrm{PE}$ is negligible. ${ }^{16,17}$ Furthermore, the two separate $\mathrm{p} K_{\mathrm{a}} \mathrm{s}$ of the polymer mixture widen the $\mathrm{pH}$ gamut where the buffer capacity is enough.

Open circuit potential $\left(E_{\mathrm{oc}}\right)$ of the SSRE was checked versus a conventional liquid junction $\mathrm{Ag} / \mathrm{AgCl} \mathrm{RE}$ (BAS model RE$5 \mathrm{~B}$ ) in $0.1 \mathrm{M}$ PBS of pH 2, 7, 12 (Figure 3). The $E_{\text {oc }}$ of the npPt 
(a)

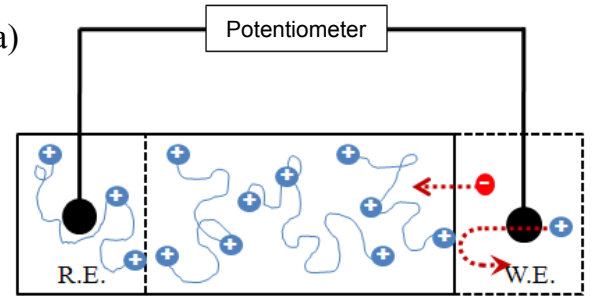

(b)

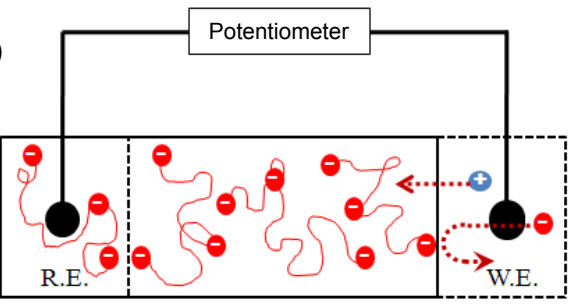

(c)

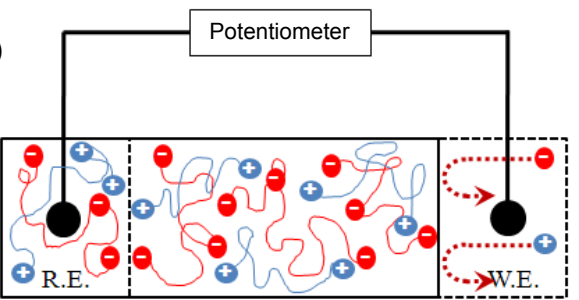

Figure 2. The working principle of $\mathrm{PE}$ junction in maintaining constant $\mathrm{pH}$ environment; (a) cationic PE (PEI), (b) anionic PE (PAA), and (c) mixed PE of opposite charge (PEI-co-PAA).

assembled with PEI (npPt/PEI) drifted continuously, as shown Figure 3(a). As for npPt/PAA, $E_{\text {oc }}$ was stable compared with that of PEI (Figure 3(b)). $E_{\text {oc }}$ of npPt/PEI-co-PAA was quickly stabilized to a constant value that was maintained at least for $1000 \mathrm{~s}$. On the whole, the $E_{\mathrm{oc}}$ of npPt/PEI was more negative than those of the npPt assembled with PAA (npPt/PAA). That is not surprising by noting that the $\mathrm{p} K_{\mathrm{a}}$ of PEI is higher than that of PAA. The effect of the $\mathrm{pH}$ of sample solution was small but not ignorable in the case of the $E_{\mathrm{oc}}$ of npPt/PEI and npPt/ $\mathrm{PAA}$. In comparison with the $E_{\mathrm{oc}} \mathrm{s}$ of npPt/PEI and npPt/PAA, $E_{\mathrm{oc}}$ of the npPt assembled with PEI-co-PAA (npPt/PEI-co-PAA) showed relatively lower variation responding to $\mathrm{pH}$ and needed shorter period for stabilization. These phenomena can be largely understood on the basis of selective ion mass transport through the $\mathrm{PE}$ junction and the $\mathrm{pH}$ range where effective buffering is valid.

Based on the results of less drifts and lower sensitivity to the $\mathrm{pH}$ change of the sample solution, PEI-co-PAA was chosen as a material for the PE junction of SSRE. Figure 4(a) shows that the $E_{\mathrm{oc}}$ of $\mathrm{npPt} / \mathrm{PEI}$-co-PAA was independent of $\mathrm{pH}$ variation between 2 and 12 and the standard deviation of the $E_{\mathrm{oc}}$ was about $5 \mathrm{mV}$. Moreover, the $E_{\text {oc }}$ of npPt/PEI-co-PAA negligibly depended on the ionic strength of the sample solution and was constant in a wide range of ion concentration $(0.1 \mathrm{M} \sim 0.1 \mu \mathrm{M})$.

Figure 5(a) shows the $\mathrm{pH}$ responses of the combined system consisting of the npPt with a polyphenol membrane (npPt/PPh) as a pH-IE and npPt/PEI-co-PAA as a SSRE. The $E_{\mathrm{oc}}$ of the $\mathrm{pH}$ sensing chip exhibited reproducible and linear responses in (a)

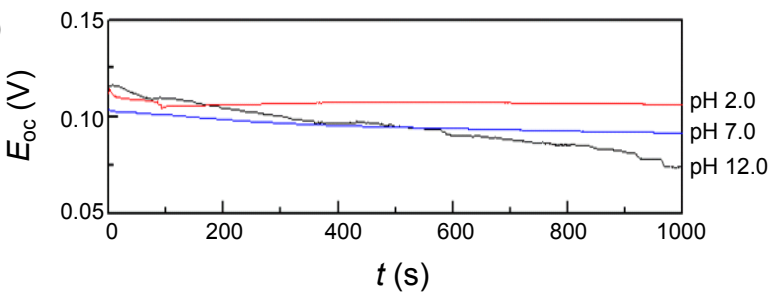

(b)

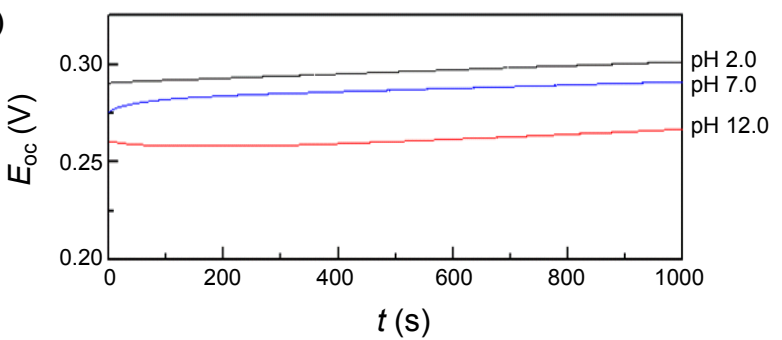

(c)

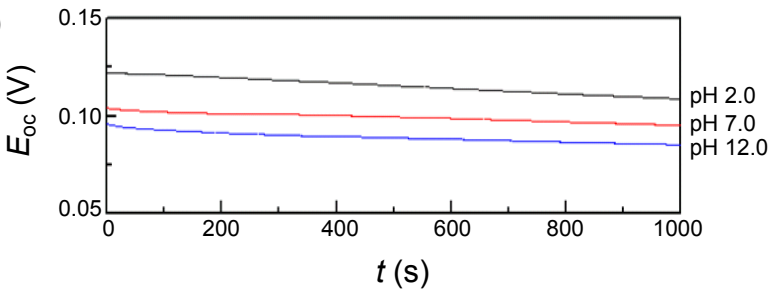

Figure 3. $E_{\text {oc }}$ of SSRE with (a) PEI, (b) PAA, and (c) PEI-co-PAA in $0.1 \mathrm{M}$ PBS (pH 2.0, 7.0, 12.0) as a function of time exposed to sample solutions.

(a)

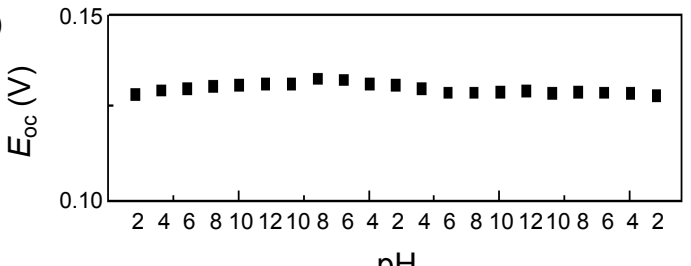

(b)

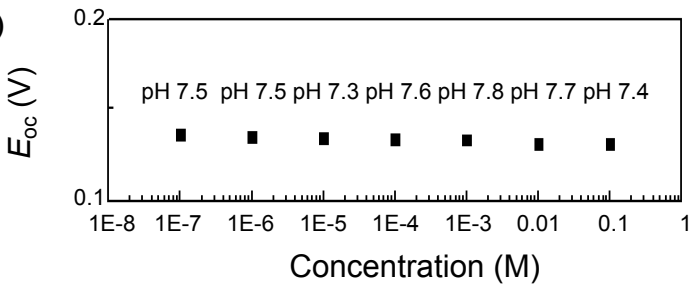

Figure 4. $E_{\mathrm{oc}}$ of SSRE versus $\mathrm{Ag} / \mathrm{AgCl}$ in (a) $0.1 \mathrm{M}$ PBS of various $\mathrm{pH}(2,4,6,8,10$, and 12) and (b) PBS solution of various ionic strength (from $0.1 \mathrm{M}$ to $0.1 \mu \mathrm{M}$ ).

the $\mathrm{pH}$ range between 2 and 12. In the previous report, ${ }^{5}$ polyphenol membrane on the npPt suppressed the interference and enhanced the performance of npPt as a pH-IE. The slope of $-45.0 \mathrm{mV}$ per $\mathrm{pH}$ of the $\mathrm{npPt} / \mathrm{PPh}$ was similar to that reported previously. ${ }^{5} \mathrm{pH}$ measurements were carried out with the proposed chip, in which a npPt/PPh (pH-IE) and a npPt/PEI-coPAA (SSRE) were integrated, for a variety of practical samples, including buffers (PBS, citrate, borate, TRIS, HEPES) and surfactant (SDS, Triton X-100) (Figure 5(b)). The responses were 
(a)

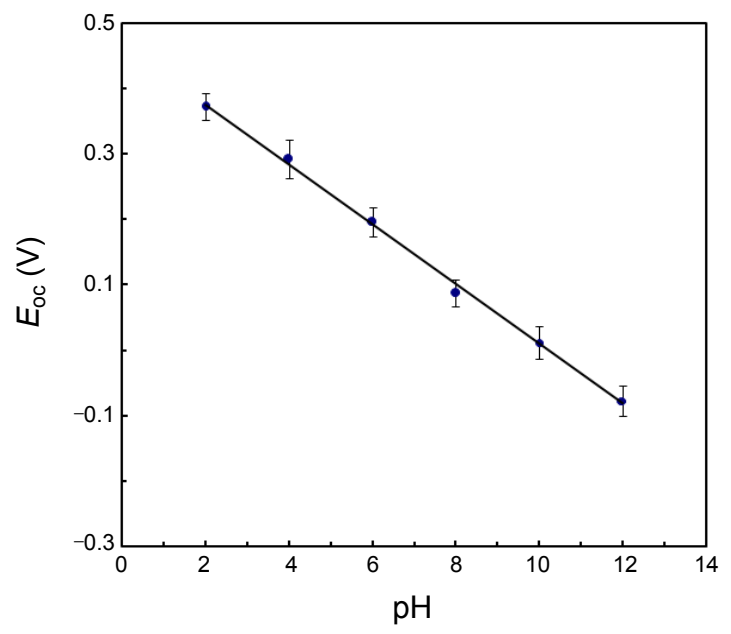

(b)

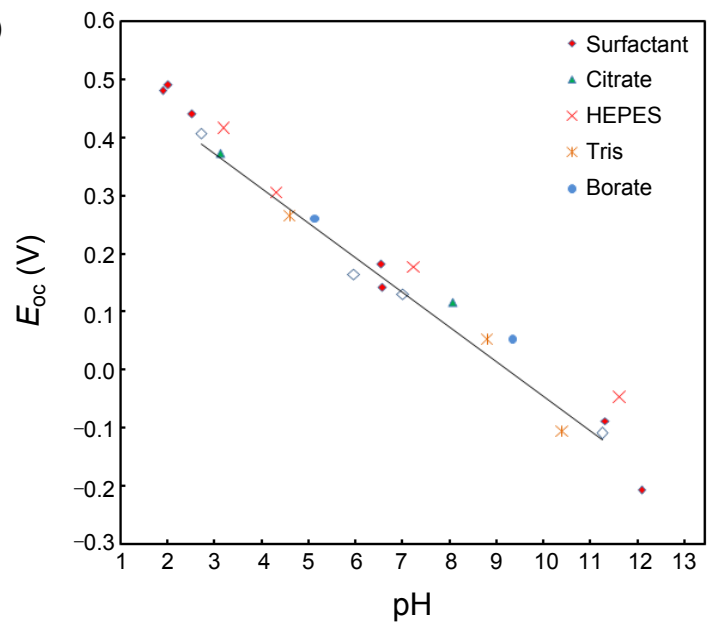

(c)

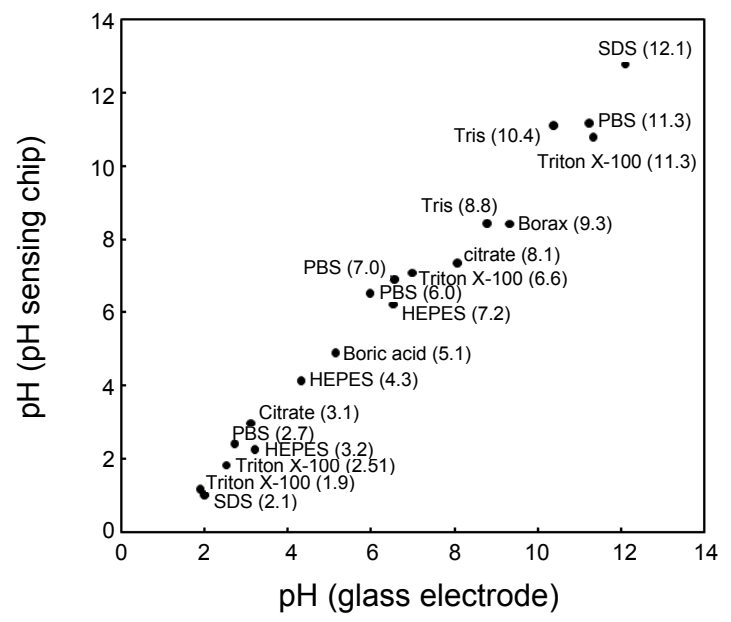

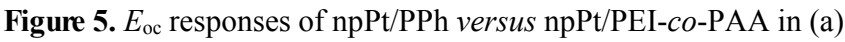
$0.1 \mathrm{M}$ PBS (pH 2, 4, 6, 8, 10, 12) $(n=5$, slope: $-45.0 \mathrm{mV}$, correlation factor: 0.998) and (b) a variety of buffers (PBS, citrate, HEPES, Tris, borate) and surfactants (SDS, Triton X-100). The $\mathrm{pH}$ values were measured by a commercial pH glass electrode (Orion, USA). (c) Correlation between $\mathrm{pH}$ values measured with a solid-state $\mathrm{pH}$ sensor and with a calibrated $\mathrm{pH}$ glass electrode.

linear and accurate with a limited deviation from the trend line. Good accuracy and linearity of the present $\mathrm{pH}$ sensor suggest the possibility of calibration-free $\mathrm{pH}$ sensing by constructing a general conversion correlation between $E_{\mathrm{oc}}$ and $\mathrm{pH}$ and then by converting the measured $E_{\mathrm{oc}}$ to $\mathrm{pH}$. The $E_{\mathrm{oc}}$ responses presented in Figure 5(b) shows a conversion correlation of $E_{\mathrm{oc}}=-59 \mathrm{pH}+$ 549 . Using this common conversion equation, the acquired $E_{\mathrm{oc}}$ data in Figure 5(b) were converted into $\mathrm{pH}$ values and checked out how they were consistent with the true values. Figure 5(c) shows the correlation between the $\mathrm{pH}$ values converted from the $E_{\mathrm{oc}}$ of the proposed solid state $\mathrm{pH}$ sensing chip and measured by calibrated $\mathrm{pH}$ glass combination electrode. The results tell that calibration-free $\mathrm{pH}$ sensing using this solid state chip system is promising.

\section{Conclusion}

A disposable solid-state $\mathrm{pH}$ sensor composed of two nanoporous Pt $(\mathrm{npPt})$ electrodes and a copolyelectrolytic (PEI-coPAA) junction was proposed and evaluated. The npPt with a polyphenol membrane (npPt/PPh) and npPt/PEI-co-PAA were employed as a $\mathrm{pH}-\mathrm{IE}$ and a SSRE, respectively. The SSRE based on PEI-co-PAA was quickly stabilized within $30 \mathrm{~s}$ and rarely affected by the chemical environments of sample solution at least for $1000 \mathrm{~s}$. These results are good enough for disposable applications. Harnessing the reliable performance of the SSRE, the proposed solid state $\mathrm{pH}$ sensor showed the capability of successful calibration-free $\mathrm{pH}$ sensing. The solid-state $\mathrm{pH}$ sensing chip in this study is mass-production friendly, easy to be miniaturized, cost effective owing to the absence of internal solution or liquid junction. Moreover, the alumina substrate and the transparent plastic cover plate are practical and cost effective alternatives to silicon substrate or glass. Consequently, this disposable and calibration-free solid state $\mathrm{pH}$ sensor is expected to make significant contributions to the biochemical, medical and analytical applications.

Acknowledgments. This work was supported by the National Research Foundation of Korea (NRF) grant funded by the Korea government (MEST) (No. 2005-2001287), the Ministry of Knowledge Economy in Korea (09MC3310, Programmable Bio-CMOS Field Effect Transistors), the Korea Research Foundation Grant funded by Korea Government (MOEHRD, Basic Research Promotion Fund) (KRF-2005-075-C00021), and the Nano/Bio Science \& Technology Program (M1053609000105N3609-00110) of the Ministry of Education, Science and Technology (MEST), Republic of Korea.

\section{References}

1. Shin, J. H.; Lee, H. J.; Kim, C. Y.; Oh, B. K.; Rho, K. R.; Nam, H.; Cha, G. S. Anal. Chem. 1996, 68, 3166.

2. Baur, J. E.; Spaine, T. W. J. Electroanal. Chem. 1998, 443, 208.

3. Marzouk, S. A. M. Anal. Chem. 2003, 75, 1258.

4. Susan, C.; Richard, P. B. Anal. Chem. 2010, 82, 878.

5. Park, S.; Boo, H.; Kim, Y.; Han, J.-H.; Kim, H. C.; Chung, T. D. Anal. Chem. 2005, 77, 7695.

6. Ha, J.; Martin, S. M.; Jeon, Y.; Yoon, I. J.; Brown, R. B.; Nam, H.; Cha, G. S. Anal. Chim. Acta 2005, 549, 59.

7. Lee, H. J.; Hong, U. S.; Lee, D. K.; Shin, J. H.; Nam, H.; Cha, G. S. Anal. Chem. 1998, 70, 3377 .

8. Kwon, N.-H.; Lee, K.-S.; Won, M.-S.; Shim, Y.-B. Analyst 2007, 
132,906

9. Vonau, W.; Enseleit, U.; Gerlach, F.; Herrmann, S. Electrochimica Acta 2004, 49, 3745.

10. Nolan, M. A.; Tan, S. H.; Kounaves, S. P. Anal. Chem. 1997, 69 , 1244.

11. Guth, U.; Gerlach, F.; Decker, M.; Oelbner, W.; Vonau, W. J. Solid State Electrochem. 2009, 13, 27.

12. Suzuki, H.; Hiratsuka, A.; Sakurai, T.; Karube, I. Sens. Actuators $B$ 1998, 46, 104.
13. Han, J. H.; Park, S.; Boo, H.; Kim, H. C.; Nho, J.; Chung, T. D. Electroanalysis 2007, 19, 786.

14. Park, S.; Lee, S. Y.; Boo, H.; Kim, H.-M.; Kim, K.-B.; Kim, H. C.; Song, Y. J.; Chung, T. D. Chem. Mater. 2007, 19, 3373.

15. Chun, H.; Chung, T. D.; Kim, H. C. Anal. Chem. 2005, 77, 2490.

16. Kim, K. B.; Han, J. H.; Kim, H. C.; Chung, T. D. Appl. Phys. Lett. 2010, 96, 143506.

17. Han, J. H.; Kim, K. B.; Kim, H. C.; Chung, T. D. Angew. Chem. Int. Ed. 2009, $48,3830$. 